\title{
Poor maternal-neonatal outcomes in pregnant patients with confirmed SARS-Cov-2 infection: analysis of 145 cases
}

\author{
Federica Di Guardo ${ }^{1}$ - Flavia Maria Di Grazia ${ }^{1}$. Luisa Maria Di Gregorio ${ }^{1}$. Elisa Zambrotta ${ }^{1}$ Grazia Carrara ${ }^{2}$. \\ Ferdinando Antonio Gulino ${ }^{3} \cdot$ Attilio Tuscano $^{4} \cdot$ Marco Palumbo $^{1}$
}

Received: 14 September 2020 / Accepted: 17 November 2020 / Published online: 3 January 2021

(c) Springer-Verlag GmbH Germany, part of Springer Nature 2021

\begin{abstract}
Purpose The coronavirus 2 (SARS-CoV-2) infection has recently spread causing millions of individuals affected globally. The raising mortality rate highlighted the necessity to identify the most susceptible populations, such as pregnant women and their fetuses, in order to protect them. Few studies have been conducted trying to identify maternal-neonatal outcomes among pregnant patients affected by COVID 19. In this scenario, this study aims to analyse poor maternal-neonatal outcomes in pregnant women affected by SARS-CoV-2 infection.

Methods This was a double-centre, 5 months retrospective analysis conducted in Italy. The study population consisted of pregnant women with confirmed SARS-CoV-2 infection assessed by Time Quantitative Reverse Transcription PCR (qRTPCR) nasopharyngeal swabs.

Results 145 pregnant women affected by confirmed SARS-CoV-2 infection were included. Among them, 116 (80\%) were symptomatic and $29(20 \%)$ were asymptomatic. Up to half of the patients $(n=111 ; 76.5 \%)$ had a past history of respiratory disease. The mean gestational age at delivery was 36 weeks \pm 5 days, while the mean maternal age was $31.5 \pm 5.63$. Reactive $\mathrm{C}$ protein (CRP) serum levels were higher than the normal range corresponding to a mean value of $56.93 \pm 49.57 \mathrm{mg} / \mathrm{L}$. The mean interval between the diagnosis of maternal COVID-19 infection and the delivery was 8.5 days. With regard to the type of delivery, the percentage of patients who delivered vaginally was higher than those who experienced a caesarean section. (74.4\% vs $25.6 \%$ ). The percentage of term birth was higher than preterm one (62\% vs $38 \%$ ). Finally, the percentages of maternal and neonatal death were found to be $5 \%$ and $6 \%$, respectively; similarly, the percentage of the infection vertical transmission was $5 \%$.

Conclusion COVID-19 infection in pregnant women seems to negatively affect both maternal and neonatal outcomes. However, it is important to emphasize that most of the cases of maternal death occurred in patients with severe symptoms and highly altered parameters related to SARS-CoV-2 infection. In the future, larger studies are warranted in order to validate these findings.
\end{abstract}

Keywords COVID-19 $\cdot$ Maternal outcomes $\cdot$ Neonatal outcomes $\cdot$ Infection

Federica Di Guardo

fediguardo@gmail.com

1 Department of Medical Surgical Specialties, Gynecology and Obstetrics Section, University of Catania, Via Santa Sofia 78, 95125 Catania, Italy

2 Department of Gynaecology and Obstetrics, San Bortolo Hospital, Viale Ferdinando Rodolfi, 37, 36100 Vicenza, Italy

3 Department of Gynaecology and Obstetrics, Azienda Ospedaliera di Rilievo Nazionale e di Alta Specializzazione (ARNAS) Garibaldi, Via Palermo, 636, 95122 Catania, Italy

4 Department od Gynecology and Obstetrics, Grande Ospedale Metropolitano - Bianchi Melacrino Morelli, Via Giuseppe Melacrino, 21, 89124 Reggio Calabria, Italy

\section{Introduction}

The severe acute respiratory syndrome caused by the Coronavirus 2 (SARS-CoV-2) has recently spread causing millions of individuals affected globally. The raising mortality rate highlighted the necessity to identify the most susceptible populations in order to protect them. Scientific evidence has recently emerged suggesting that pregnant women and their foetuses are vulnerable category at high risk for SARSCoV-2 infection. Moreover, the virus may determine poor outcomes both in infected pregnant patients and in their fetuses/newborns with a rate of admission to intensive care 
units (ICU) of up 35\% [1, 2]. Explanation for SARS-CoV-2 susceptibility in pregnant women relies on anatomical changes occurring during gestation: the increased dimension of the thoracic cage transverse diameter as well as the elevation of the diaphragm level may cause detrimental effects on maternal hypoxia tolerance [3, 4]. Moreover, the modifications of lung volume and the increased vasodilatation might improve mucosal edema and secretions in the upper respiratory tract. With regard to the immunology, it seems that during pregnancy there is an increased risk to contract infections caused by intracellular organisms, such as viruses, due to the alteration of the cell-mediated immunity [5]. Considering the psychological/mental health aspect, it is important to highlight that pregnant women hospitalized in high-risk departments are described to be at major risk for depression [6]. In line with this, pregnant patients affected by COVID-19 infection with hospitalization in high-risk pregnancy units reported a similar trend towards depression when compared to those not affected [7]. On the other hand, recent evidence reported a lower risk for post-partum depression in women delivering during the COVID-19 pandemic when compared to those patients not delivering during the pandemic [8]. Considering fetuses and newborns, the higher susceptibility to SARS-CoV-2 may be explained by the immaturity of their innate and adaptive immune systems [9]; furthermore, cytokines pathways alteration as well as dysregulation of complement cascade might determine detrimental outcomes on neurological development and brain functions [10]. In this view, the hypothesis of a SARS$\mathrm{CoV}-2$ vertical transmission has been recently proposed and investigated, representing a topic of particular interest for both gynecologists and pediatricians [11]. In this scenario, this study aims to analyse severity of maternal-neonatal outcomes in pregnant women affected by SARS-CoV-2.

\section{Material and methods}

\section{Study design}

This was a retrospective, double-centre cohort study including pregnant symptomatic and asymptomatic women with confirmed SARS-CoV-2 infection attending the Department of Gynaecology and Obstetrics of two Italian tertiary referral hospitals from March 2020 to July 2020.

\section{Overview on COVID-19 pandemic in Italy}

The drastic spread of COVID-19 in Italy prompted adequate measures for the containment of its transmission [12]. On April 1, 2020 a total number of 110.574 documented cases and 13.155 documented deaths related to SARS-CoV-2 infection were reported, with an increasing trend during the subsequent days and a mortality rate of up to $10 \%$ [13]. However, it is of paramount importance to explain the reasons for such a high mortality rate in the Italian general population in order to develop optimal management strategies to face the future. The mean Italian population age may be identified as one of the contributing factors; indeed, Italy has the most elderly population in Europe and the second most elderly population in the world after Japan. The median age of Italian people infected with SARS-CoV-2 infection, and who subsequently died, has been reported to be around 70-75 years, while the average age of patients requiring critical care-support is around 65 years [14]. Moreover, COVID-19 morbidity and mortality strongly depend on the presence of concomitant respiratory diseases. In this context, Italy reported an elevated percentage of patients with a history of severe smoking and high rates of chronic obstructive pulmonary disease as well as cardiovascular/ischemic affections [15]. With regard to the Italian female general population, the mortality rate is reported to be around 5.8\% [16]. Nevertheless, assessing the mortality rate in young pregnant women is to date particularly challenging due to the poor data reported on the topic so far.

\section{Study population}

The study population consisted of pregnant symptomatic and asymptomatic Italian women with confirmed SARS-CoV-2 infection. The SARS-CoV-2 maternal infection was assessed by results of positive Time Quantitative Reverse Transcription PCR (qRT-PCR) nasopharyngeal swabs. Female patients with physiologic pregnancies were included in the study. Moreover, patients with previous/current history of obstetrics pathologies (such as preeclampsia and/or gestational diabetes) under control as well as those with physiologic twin pregnancies were also included in the analysis. Conversely, monochorial monoamniotic twin pregnancies and multiple pregnancies were excluded due to their high risk of complications.

\section{Main outcomes measures}

The aim of the presented study was to investigate poor maternal-neonatal outcomes associated with the presence of infection in pregnant patients affected by SARS-CoV-2. Primary outcome was maternal death while secondary outcomes were neonatal death, vertical infection transmission and preterm birth.

\section{Vertical transmission assessment}

The possibility of vertical transmission was assessed through COVID-19 RT-PCR test on amniotic fluid, placenta and cord blood. 


\section{Data presentation}

Continuous data are presented as mean \pm standard deviation (SD) and categorical data are described by number of cases and percentages. Analysis was performed using the Stata/SE v.14.1 software, StataCorpLP, USA.

\section{Results}

\section{Patient baseline characteristics}

In total, 145 pregnant women affected by confirmed SARS$\mathrm{CoV}-2$ infection were included. Among them, the major percentage were at the third trimester of pregnancy $(92.5 \%)$ while $7.5 \%$ were at the second trimester. Furthermore, 116 (80\%) manifested symptoms of COVID-19 infection (fever, cough, pneumonia) and $29(20 \%)$ were asymptomatic. Up to half of the patients $(n=111 ; 76.5 \%)$ had a past medical history of respiratory diseases. The mean interval between the diagnosis of maternal COVID-19 infection and the date of delivery was $8.5 \pm 2$ days. With regard to the type of delivery, the percentage of patients who delivered vaginally was higher than those who experienced a caesarean section. ( $74.4 \%$ vs $25.6 \%)$. The mean gestational age at delivery was 36 weeks \pm 5 days, while the mean maternal age was $31.5 \pm 5.63$. The Reactive $C$ protein (CRP) serum levels were $56.93 \pm 49.57 \mathrm{mg} / \mathrm{L}$. Patient baseline characteristics are displayed in Table 1 .

\section{Maternal-neonatal outcomes}

Maternal-neonatal outcomes are reported in Table 2. The percentage of term birth was higher than preterm one $(62 \%$ vs $38 \%$ ). Cases of maternal death were 7 , corresponding to $5 \%$. Similarly, the number of women admitted to ICU was $8(5.5 \%)$; all of them were ventilated and intubated. The percentage of neonatal death and infection vertical transmission was $6 \%$ and $5 \%$, respectively (Table 2 ). Detailed data on neonatal deaths cases are illustrated in Table 3.

\section{Discussion}

The results of our large retrospective series highlighted a percentage of maternal death of $5 \%(n=7)$. Considering the totality of maternal death cases, all women were affected by severe COVID-19 infection with high fever, haemoptysis and pneumoniae. Moreover, the majority of them (4/7) had a past history of respiratory disease, CRP levels above the normal range and diminished platelets counts. All the cases of death were intubated, recovered in the ICU and died of acute respiratory insufficiency. With regard to the neonatal
Table 1 Patients' baseline characteristics of 145 women included in the study

\begin{tabular}{ll}
\hline Baseline characteristics & \\
\hline Age & $31.5 \pm 5.63$ \\
Mean (SD) & \\
Gestational age (week \pm days) & $36 \pm 5$ \\
Mean (SD) & \\
CRP (mg/L) & $56.93 \pm 49.57$ \\
Mean (SD) & \\
Interval diagnosis-delivery (days) & $8.5 \pm 2$ \\
Mean (SD) & \\
History of respiratory disease & $111(76.5 \%)$ \\
Yes & $34(23.5 \%)$ \\
No & $116(80 \%)$ \\
Symptoms & $29(20 \%)$ \\
Yes & \\
No & $11(7.5 \%)$ \\
Pregnancy trimester at admission & $134(92.5 \%)$ \\
II & \\
III & $4(5.8 \%)$ \\
Pregnancy trimester at delivery & $141(94.2 \%)$ \\
II & $108(74.4 \%)$ \\
III & $37(25.6 \%)$ \\
Type of delivery & \\
Vaginal & \\
Casearian section & \\
\hline
\end{tabular}

Values are expressed as mean (SD); Values are expressed as number $(n)$ and percentage (\%); Interval Diagnosis-Delivery: Interval between the diagnosis of maternal COVID-19 infection and the date of delivery in days

$C R P$ reactive $\mathrm{C}$ protein $(\mathrm{mg} / \mathrm{dL})$

Table 2 Maternal and neonatal outcomes among 145 women included in the study

\begin{tabular}{lc}
\hline Maternal and neonatal outcomes & \\
\hline Preterm birth & \\
Yes & $55(38 \%)$ \\
No & $90(62 \%)$ \\
Maternal admission to ICU & $8(5.5 \%)$ \\
Yes & $137(94.5 \%)$ \\
No & \\
Maternal death & $7(5 \%)$ \\
Yes & $138(95 \%)$ \\
No & \\
Neonatal death & $9(6 \%)$ \\
Yes & $136(94 \%)$ \\
No & \\
Vertical transmission & $7(5 \%)$ \\
Yes & $138(95 \%)$ \\
No &
\end{tabular}

Values are expressed as number $(n)$ and percentage (\%) 
Table 3 Extensive evaluation of the neonatal death cases

\begin{tabular}{|c|c|c|c|c|c|}
\hline $\begin{array}{l}\text { Gestational } \\
\text { age at deliv- } \\
\text { ery }\end{array}$ & Type of delivery & Apgar & Weight at birth & Death causes & Maternal outcome \\
\hline $36+2$ & Caesarean section & 1 & $2731 \mathrm{~g}$ & Tachycardia, internal, haemorrhage, DIC & Alive, no admission to ICU \\
\hline 34 & Caesarean section & 2 & $2472 \mathrm{~g}$ & Acute foetal distress & Alive, no admission to ICU \\
\hline $34+5$ & Caesarean section & 3 & $2790 \mathrm{~g}$ & Acute foetal distress & Alive no admission to ICU \\
\hline $30+4$ & Caesarean section & 2 & $1833 \mathrm{~g}$ & Acute foetal distress & Tachypnea, fever alive, admission to ICU \\
\hline $35+2$ & Caesarean section & 3 & $2681 \mathrm{~g}$ & Acute foetal distress, sepsis, DIC & Alive no admission to ICU \\
\hline $30+3$ & Caesarean section & 2 & $1798 \mathrm{~g}$ & Acute foetal distress & Alive, ARDS admission to ICU \\
\hline 28 & Vaginal delivery & 1 & $995 \mathrm{~g}$ & Acute foetal distress & $\begin{array}{l}\text { Alive, ARDS, ARF intubated, admission } \\
\text { to ICU }\end{array}$ \\
\hline 24 & Caesarean section & 1 & $570 \mathrm{~g}$ & Acute foetal distress & $\begin{array}{l}\text { Alive, ARDS, intubated, admission to } \\
\text { ICU }\end{array}$ \\
\hline 28 & Caesarean section & 1 & $1039 \mathrm{~g}$ & Acute foetal distress, coagulopathy & $\begin{array}{l}\text { Alive, ARDS, intubated, admission to } \\
\text { ICU }\end{array}$ \\
\hline 36 & Caesarean section & 4 & $2445 \mathrm{~g}$ & Acute foetal distress & Alive, no admission to ICU \\
\hline
\end{tabular}

DIC Disseminated Intravascular Coagulation, ICU Intensive Care Unit, ARDS Acute Respiratory Distress Syndrome, ARF Acute Renal Failure

death, its percentage was $6 \%(n=9)$. The most common cause of death in newborns was acute foetal distress. However, it is important to highlight that Disseminated Intravascular Coagulopathy (DIC) and sepsis were also reported among the death causes. The occurrence of the infection's vertical transmission was assessed for all the neonates via RT-PCR test on amniotic fluid, cord blood and placenta, collected immediately after birth. COVID-19 vertical transmission was identified in $5 \%$ of foetuses $(n=7 ; 2$ among death neonates and 5 among alive ones). The percentage of preterm birth was lower than at term one (38\% vs $62 \%$ ). Interestingly, we noted that the mean serum CRP levels were higher than normal range. Furthermore, the mean gestational age at delivery was 36 weeks \pm 5 days.

Few studies have investigated poor maternal-neonatal outcomes, including death, occurring both in mothers and newborns who developed COVID-19 infection [17, 18]. Our study results identified a consistent percentage of maternal/neonatal death in pregnant patients affected by COVID-19 and respective neonates. The fact that four out of seven women who died had experienced severe symptomatic COVID-19 infection, CRP high serum levels and low platelet counts deserves further consideration, possibly underlining COVID-19 pathogenetic mechanisms still unknown. Although maternal deaths occurred due to acute respiratory insufficiency in patients with relevant COVID19 symptoms, the poor evidence reported on the topic so far has slightly highlighted the association between maternal death and the status of severe COVID-19 symptomatic patient [19]. Considering CRP serum concentration, inflammatory markers have been reported to be often substantially elevated in patients with severe COVID-19 as result of selfperpetuating, and tissue-damaging inflammatory activity
[20, 21]. Recent studies on COVID-19 infection have identified a strong association between the status of critical illness and high serum levels of CRP [22]. In line with this, our analysis showed serum CRP levels above the normal range in pregnant patients affected by SARS-CoV-2. Conversely, our results are in contrast with the ones reported by Wang D et al., who found below-reference serum CRP concentrations in pregnant women affected by COVID-19 pneumonia [23]. However, the small sample size of the aforementioned study, including only 16 pregnant patients affected by COVID-19 pneumonia, represents an important bias to be considered when interpreting the results. With regard to the rate of premature delivery, our data are partially discordant with those reported so far in literature, which revealed a higher percentage of preterm delivery in confirmed COVID-19 patients [24]. Although our study reported a higher percentage of at term births (62\%), preterm birth occurred in $38 \%$ of the cases. This information is consistent with the mean gestational age at delivery which corresponds approximately to 36 weeks \pm 5 days of gestation. Moreover, it is not concordant with the rate of preterm delivery in the general pregnant population accessing our institutions (8.75\%). Finally, considering the delivery modality, our results are in contrast with those reported by $\mathrm{Na} \mathrm{Li}$ et al. who found a higher percentage of caesarean sections in COVID-19 patients; however, this study has a strong limitation as COVID-19 infection was considered one of the indications for caesarean delivery in the hospital where the study was conducted [19]. In this scenario, our study results may rely on the suggestion reported by Favre et al. in the "Guidelines for pregnant women with suspected SARS-CoV-2 infection", proposing that vaginal delivery could be considered for the benefit of patients, when labour rooms are properly equipped for 
airborne precautions [25]. Since our study showed a higher percentage of spontaneous delivery than caesarean section, we assume that Favre et al. recommendations has been probably well received. Furthermore, this result is consistent with the rate of spontaneous delivery in our institutions (60.1\%). The strength of our study relies on its design, including a large sample size of pregnant women affected by COVID19 infection. Moreover, it descriptively reported the effects of COVID-19 infection on maternal-neonatal outcomes. Despite the robust design of our study, limitations do exist due to its retrospective nature which represents itself a risk of bias. Therefore, caution has to be applied when interpreting the results.

In conclusion, COVID-19 infection in pregnant women seems to negatively affect both maternal and neonatal outcomes. However, it is important to empathise that most of the cases of maternal death occurred in patients with severe symptoms and highly altered parameters due to SARSCoV-2 infection. In the future, larger studies are warranted in order to validate these findings.

Acknowledgments The authors thank FMDG and LMDG for the data collection.

Author contributions FDG designed the study and wrote the manuscript. FMDG, LMDG and GC are responsible for data collection. EZ, FAG, AT, FDG and MP critically commented and substantially revised the manuscript. All authors participated in drafting the manuscript and approved the final version.

Funding This work was not supported by any grant or other form of funding.

\section{Compliance with ethical standards}

Conflict of interest The authors declare that any conflicts of interest are present about this study.

Ethical approval This retrospective study was conducted in accordance with ethic guidelines for retrospective studies of our institution Ethics Committee of University of Catania, Policlinico-San Marco Hospital. Patients' anonymity was guaranteed. The principles of the Helsinki Declaration form were respected.

Informed consent Informed consent was obtained from all individual included in the study.

\section{References}

1. Wong SF, Chow KM, Leung TN, Ng WF, Ng TK, Shek CC, Lai ST (2004) Pregnancy and perinatal outcomes of women with severe acute respiratory syndrome. Am J Obstet Gynecol 191(1):292-297

2. Alfaraj SH, Al-Tawfiq JA, Memish ZA (2019) Middle East Respiratory Syndrome Coronavirus (MERS-CoV) infection during pregnancy: report of two cases and review of the literature. J Microbiol Immunol Infect 52(3):501-503
3. Goodnight WH, Soper DE (2005) Pneumonia in pregnancy. Crit Care Med 33(10):S390-S397

4. O'Day MP (1997) Cardio-respiratory physiological adaptation of pregnancy. In: Seminars in perinatology, vol. 21, no. 4. WB Saunders, Philadelphia, PA, pp 268-275

5. Nelson-Piercy C (2020) Handbook of obstetric medicine. CRC press.

6. Hermon N, Wainstock T, Sheiner E, Golan A, Walfisch A (2019) Impact of maternal depression on perinatal outcomes in hospitalized women - a prospective study. Arch Women's Mental Health 22(1):85-91

7. Sade S, Sheiner E, Wainstock T, Hermon N, Yaniv Salem S, Kosef T, Pariente G (2020) Risk for depressive symptoms among hospitalized women in high-risk pregnancy units during the COVID-19 pandemic. J Clin Med 9(8):2449

8. Pariente G, Broder OW, Sheiner E, Battat TL, Mazor E, Salem SY, Wainstock T (2020) Risk for probable post-partum depression among women during the COVID-19 pandemic. Arch Women's Ment Health. https://doi.org/10.1007/s00737-020-01075-3

9. van Well GT, Daalderop LA, Wolfs T, Kramer BW (2017) Human perinatal immunity in physiological conditions and during infection. Mol Cell Pediatr 4(1):4

10. Tsafaras GP, Ntontsi P, Xanthou G (2020) Advantages and limitations of the neonatal immune system. Frontiers in Pediatrics 8:5

11. Chan GJ, Lee AC, Baqui AH, Tan J, Black RE (2013) Risk of early-onset neonatal infection with maternal infection or colonization: a global systematic review and meta-analysis. PLoS Med 10(8):e1001502

12. Remuzzi A, Remuzzi G (2020) COVID-19 and Italy: what next? Lancet 395(10231):1225-1228

13. Istituto Superiore di Sanità. ISS. Accessed November 3,2020 http://www.cuore.iss.it/indagini/CuoreData

14. Boccia S, Ricciardi W, Ioannidis JP (2020) What other countries can learn from Italy during the COVID-19 pandemic. JAMA internal medicine, 180(7):927-928

15. Organization for Economic Co-Operation and Development. OECD. Stat database. Accessed November 3, 2020. https://stats .oecd.org/

16. Di Stadio A, Ricci G, Greco A, de Vincentiis M, Ralli M (2020) Mortality rate and gender differences in COVID-19 patients dying in Italy: a comparison with other countries. Eur Rev Med Pharmacol Sci 24(8):4066

17. Zaigham M, Andersson O (2020) Maternal and perinatal outcomes with COVID-19: a systematic review of 108 pregnancies. Acta Obstet Gynecol Scand 99(7):823-829

18. Della Gatta AN, Rizzo R, Pilu G, Simonazzi G (2020) COVID19 during pregnancy: a systematic review of reported cases. Am J Obstet Gynecol 223(1):36-41

19. Li N, Han L, Peng M, Lv Y, Ouyang Y, Liu K, Yang L (2020) Maternal and neonatal outcomes of pregnant women with COVID-19 pneumonia: a case-control study. Clin Infect Dis 71(16):2035-2041

20. Ruan Q, Yang K, Wang W, Jiang L, Song J (2020) Clinical predictors of mortality due to COVID-19 based on an analysis of data of 150 patients from Wuhan, China. Intensive Care Med 46(5):846-848

21. Chen N, Zhou M, Dong X, Qu J, Gong F, Han Y, Yu T (2020) Epidemiological and clinical characteristics of 99 cases of 2019 novel coronavirus pneumonia in Wuhan, China: a descriptive study. The Lancet 395(10223):507-513

22. Channappanavar R, Perlman S (2017) Pathogenic human coronavirus infections: causes and consequences of cytokine storm and immunopathology. In: Teijaro J (ed) Seminars in immunopathology, vol 39, No. 5. Springer, Berlin, Heidelberg, pp 529-539

23. Wang D, Hu B, Hu C, Zhu F, Liu X, Zhang J, Zhao Y (2020) Clinical characteristics of 138 hospitalized patients with 2019 
novel coronavirus-infected pneumonia in Wuhan, China. JAMA 323(11):1061-1069

24. Chen H, Guo J, Wang C, Luo F, Yu X, Zhang W, Liao J (2020) Clinical characteristics and intrauterine vertical transmission potential of COVID-19 infection in nine pregnant women: a retrospective review of medical records. The Lancet 395(10226):809-815

25. Favre G, Pomar L, Qi X, Nielsen-Saines K, Musso D, Baud D (2020) Guidelines for pregnant women with suspected SARSCoV-2 infection. Lancet Infect Dis 20(6):652-653
Publisher's Note Springer Nature remains neutral with regard to jurisdictional claims in published maps and institutional affiliations. 